\title{
Flexible Control System for Robot Assembly Automation
}

\author{
Li-Chen Fu $u^{1,2}$ and Larry Jann ${ }^{1}$ \\ Dept. of Computer Science \& Information Engineering ${ }^{1}$ \\ Dept. of Electrical Engineering ${ }^{2}$ \\ National Taiwan University
}

\begin{abstract}
In this paper, we propose an object-oriented model for a control system architecture of robotic assembly automation and extend this model to all event driven automation system. Based on this model, we develop a systematic procedure by means of EDAK claslib (Event Driven Automation Kernel Class Library ) to support the users to easily implement any event driven automation system. The by-product is to allow system designer to easily expand an existing system or integrate several event driven automation systems which are all supported by EDAK control system.
\end{abstract}

\section{Introduction}

Mass-production assembly lines were first introduced by the Ford Motor Company [2]. Over the ensuing decades, specialized machines have been designed and developed for high-volume production of mechanical and electrical parts. Until recent years, the robot application was developed for large quantity production. To upgrade the robot intelligence needed in a varying task environment, various sensors need to be incorporated first. Besides this powerful sensing, integration of planning, decision making capabilities, analysis devices, and robust controllers has to be introduced. Such integration needs to be supported by a well designed computer architecture to meet the computational requirement of each subsystem. An intelligent robotic assembly system consists of several subsystems capable of providing dynamic interactions with the enviromment in order to accomplish a task properly.

In this paper, we propose an object-oriented model for a control system architecture of robotic assembly automation and extend this model to all event driven automation system. Based on this model, we develop a systematic procedure by means of EDAK classlib (Event Driven Aulomation Liernel (lass Library) to support the users (system designers) to easily implement any event driven automation system. The by-product of this control system is to allow system designers not to worry about making any change to the overall system architecture even in face of system expansion, i.e., the present system can be upgraded easily from any system expansion. Moreover, it will bestraightforward to integrate any other event driven automation systems which are supported by EDAK control system.

\section{Robotic Assembly Workstation}

In a typical assembly factory, there is usually a stationary and well-defined place for the assembly and inspection tasks, which is usually called "assembly workstation". Some critical equipment should be traken into consideration when configuring an assembly workstation [5], which includes: Robot, End Effector, Part Feeder, Transfer Mechanism, assembly Fixture and Sensors.

\subsection{Control Scheme for Robotic Assem- bly System}

Robotic assembly systems are usually controlled by a scheme with hierarchical structure which is divided into five levels, namely, task planning, subtask decomposition, trajectory planning, trajectory execution, and manipulation (as depicted in Fig. 2.1). Such a hierarchical control scheme may be implemented in a distributed environment [7], or can be employed in some centrally controlled cell.

\subsection{Problem Formulation}

Software design of the programming/planning and task execution for an advanced robot system may be viewed as a problem of defining two taxonomies, namely, planning and execution, and the relationships between them [17]. The requirements for these two taxonomies are, to a large extent, conflicting. Task planning requires an operational taxonomy, in which the actions to be performed are classified Classification of actions is beneficial in planning since it leads to pruning of the set of operators to be considered in constructing a plan, and facilitates the construction of the generic "exception handling operations" $[18]$

On the contrary, the run-time environment for task execution benefits from the imposition of a fumctional taxonomy, in which the physical objects to be operated upon are classified. Functional classification provides the benefits of modular development for large, and durable, software system [19].

To sum up, a systematic and convenient utility which can help the system designers to build a robust, efficient, expandable, and dynamically reconfigurable automation system is then developed in this paper. It is called EDAK (Event Driven Automation Kernel) system, and will be detailedly described in the following section. 


\section{Flexible Control System}

This subsection will focus on the model of this event driven automation environment using objectOriented method. The basic object here is the Handler (which will be described later). In another words, this is a handler based automation system.

Consider an event driven automation environment, where normally there will be four basic entities as Control Kermel, Application Task, Ilandler, and Planner ( see Fig. 3.1). A handler is a software process or a hardware piece of equipment which provides necessary functionalities for the automation system. For example, image process, motion planning process, and simulation process are software ones, whereas robot, $\mathrm{AGV}$, mobile robot, conveyor belt, buffer, camera, and optical switches are hardware ones.

A control kernel is a process that functions as an intermediary among handler processes. The purpose of a control kernel is to provide an enviromment in which a handler process can rum smoothly and conveniently. Therefore, its primary goal is to make the automation system convenient to use. In fact, we usually want our developed automation system to operate in an efficient manner so as to serve the need of real-time response. (onsequently, a control kemel should provide an excellent back-bone to the automation system so that it can meet the demand.

\subsection{Handler}

According to the task execution property, handlers can be classfied into two types: sequential handler and parallel handler. A sequencial handler is a sequencial task executer, which can not handle more than one task in a duty cycle. That is, it accepts the next command only when it finishes the current one. Nevertheless, the parallel handler is able to perform multiple tasks which dispatched from other handlers. In addition, almost all hardware equipment, such as robots, are sequencial handlers.

\subsubsection{Handler Hierarchy}

If a handler $\mathrm{A}$ is directly managed by another luandler $B$, then we say that $A$ is a Child Handler of $B$, or $B$ is a Parent Handler of A. We illustrate the relationship) between them in Fig. 3.2.

For instance, an ATC (automatic tool changer) is a child handler of its corresponding robot handler which seems to be quite natural as well. Specially, if a handler is dedicated to another handler, then the former is a child handler of the latter. In the sequel, we should realize that EDAK can also be a parent, handler of some other liandler, for it can be viewed as a handler.

Considering another situation, if a device handler is shared by two or more handlers, then it owns multiple parent handlers (see left side of Fig. 3.3). It should execute the commands from the multiple parent handlers, that will cause a coordinating problem among those parent handlers in which the child one is a sequencial handler. That is, if the child handler is a parallel handler, that problem will not appear since the natural parallel processing capability of it. But if it is not parallel one, then an appropriate coordinating procedure is necessary for those parent handlers.

The technique for solving the aforementioned problem will be described as follows. Mainly, its procedure is to upgrade the child handler as a sibling one of those parent handlers so that this problem is changed to a cooperation problem which call fluen be managed by the original grandparent handler (see right side of Fig. 3.3).

\subsubsection{Communication Transparency and Agent Process}

An automation system sometimes can be considered as a distributed system. When a number of sites are connected to one another in that system by a communication network, the users at different sites will have the opportunity to exchange information. But it is difficult to ask all pieces of automated equipment to run a communication network using a single scheme. To solve this lack of the communication transparency, each of the child handlers of EDAK should have a standard interface to communicate with one other. This may be achieved via the socket networking which is the most popular communication technique so far. If a handler's control process is to be running on a socket network environment, such as Unix operating system platform, then we need to build a standard interface for it. Therefore, it does not need another agent process; otherwise, it must have an agent process for interface between the control process of the handler and the socket communication environment, which will then pass the information from any other handlers or the kernel to this control process via specific communication medium. (see Fig. 3.4)

On the contrary, if none of all the parent handlers of a child handler is EDAK, the child one will no longer need a standard interface or agent process at all.

\subsection{Control Kernel : EDAK}

Here the control kernel plays the role of governing all handlers to follow reasonable flow to achieve a smooth working environment. Since handlers may include manipulators, planners, and event detectors, the control kernel may be considered as a resource allocator. An automation system has many resources that may be required to solve a problem: robot utilization, part flow, cooperation among handlers, collision detection and avoidance among handlers, etc. The control kernel functions as the manager of these resources and allocates them to specific tasks and operators as necessary. Since there may be many, possibly conflicting, requests for resources, the control kernel with the planner must decide which requests are taken into account for allocating resources so that the automation system will work efficiently and fairly.

As a result, in this paper we develop a control kernel, called EDAK (Event Driven Automation liernel), which consists of three components: shell, dalabase, and main body, as shown in Fig. 3.5, respectively, explained below.

- Shell: It is the message entry of the kernel from any possible senders. According to the type of individual message, the kernel then issues suitable responses.

- Main body: Properly managing the commands and resources is the major job of main body.

- Database: Since the system requires complete information to help it make an appropriate decision, the database needs to record the desired information.

\subsection{Application Task : Command}

A task is just a command when viewed from the Conlrol ficrnol. In general, a task needs certain 
resources, such as manipulating devices and target parts. These resources are either assigned to the task when it is created, or allocated to it while it is running. The operators, handlers or planners generate tasks in demand, then the shell put them into a command queue, and then these tasks wait for being fetched to execute.

\subsubsection{Command Attribute}

The tasks in general can be classified into two level command: macro level and micro level commands. The former one, macro level command, is a combination of micro level commands. The objective to design these two types of command is simply for convenience and flexibulity.

In addition, these commands are also categorized to have two types of attributes: blocking and nonblocking. When a command is executed, it will try to allocate the demanded resources. If the allocation succeeds, the command is just issued out. Otherwise, the fate of this command will depend on its attribute. If it belongs to the non-blocking type, it and all of its successive commands will all be deleted and then an error message will be sent to the user who created it If it belongs to the blocking type, it means that this command can wait for the resource for a longer while and, therefore, it will be put, into the waiting queue of that resource. While the resource is relcased, all of the commands in the waiting quene of the resource will be put into the command queue again and that resource will be attempted one nore time.

\subsubsection{Task State}

As a task is being executed, it will change from one slate to another, where each state is defined by the properties of the current activity. Each task may mudergo different five states as described below (see Fig. $3.6)$ :

- Ready: It means that the task is waiting to be executed, which is already put, into command queue by control kernel via shell interface.

- Executing: It smmbolizes that the task is being executed. If the task is a micro level command, it will try to allocate resources. If it succeeds, then this command is issued out to the handler; otherwise, the macro level command then spawns a sequence of micro level commands which are connected among thenselves via an AOV (activate on vertex, as shown in Fig. 3.7) network relationships, and put the root commands of the network into the command queue for further execution.

- Waiting: If a micro level command can not acquire its desired resources when it was previously in the executing state but its attribute belongs to the blocking type then the control kermel will put it into the waiting gueue.

- Rumming: A command is just being issued out to the corresponding handler and is waiting for a report of the execution results.

- EndWorking: This command is terminating such as modifying system state or releasing re source, after the execution result was reported from the corresponding handler. An important command termination is to execute the successive commands by placing them into the command queue.

\subsubsection{Two-Step Command}

Here, we develop a two-step command (see Fig. 3.8) which proceeds with two member functions: execute and end Work corresponding to the executing and the endWorking states, respectively, as described below in detail:

- execute(): Before a given command (task) is executed, it first requires necessary resources allocation, such as robot, part, etc. Furthermore, the cornmand needs to extract some desired parameters for its handler from the database. When the resource allocation is finally done, this command will be issued out to the handler.

- endWork(): When this command is done by the handler, it sends a Result Report message back which contains the executing result of this command. Then, according to the result, we can undertake some closing operation. For example, after a successful execution, the resource is released and the database is updated accordingly, and its successive command follows suit.

\subsection{Planner}

The planner is a special handler of control kernel, which is also a crucial one. In fact, one can have multiple planners to form a decision making group. It depends on how one wants to construct such intelligent mechanism and to exhibit the flexibility of this system. If a EDAK system lacks the planner handler, the whole system becomes quite rigid in terms of the activity flows.

\section{System Properties with EDAK}

A complex automation system usually combines a lot of pieces of equipment. Any addition of a machine device, which is to be integrated into the system, will need a number of procedures which sometimes require non-negligible efforts and considerable amount of time. In this section we propose a technique to solve this problem - the Registration Procedure (see Fig. 4.1). This approach is just to partition the handler functionality into several small pieces. Each of these pieces should be well-defined and can be uniformly described, with carefully defined inputs, outputs, and function.

When EDAK is booting, none of handlers will be resiclent. But when EDAK is ready, then all of the handlers are booting in some random sequence. At the first step, the handler diagnoses and initializes itself. If everything is all right and is standby, the handler tries to register on EDAK. In fact it transfers a "landler registration" command to EDAK via communication medium. In this command, there are lots of parameters and information to fully describe the role or the characteristics of this handler so that the EDAK can appropriately manage it. EDAK will create an ambassador object and record all the received information in it, such as communication channel, supporting functions, performance, and so on. Finally, if the registration procedure is successfully done on control kernel, it will reply a message inchuding a unicue series number, like an identification number in EDAK, back to the handler. Also, EDAK will identify the specific handlers by use of the series number wherever there is a message sent in.

Suppose that a handler has malfunctioned, it can quit out the system actively or passively. The quitout procedure is similar to the registration one. The 
control kernel will remove the corresponding ambassador object of the handler and then the system still operates properly as usual. When a failed handler is repaired, it must notify the kernel that it is up again. It is quite easy that by the registration procedure again, $E D A K$ can dynamically record and maintain the whole available handlers at, any time.

Moreover, we propose another technique - "function registration", which cause the handler's functionalities can be online modification.

\subsection{Starvation and Deadlock Avoidance}

As mentioned before, if a command which belongs to the blocking type can not acquire its desired resources, it will be pending on the kemel for a long time. But how can we avold the starvation situation or the deadlock one?

$E D A K$ always receives a tick signal once every constant period of time. When obtaining a tick, EDAK will increase the waiting time and check the elansed ticks of each current commands in the system. If the time bound of the checked command is forener, nothing will be done on it. Otherwise, if the number of elapsed ticks exceeds its time bound, EDAK will remove this cormmand and reply an error message to the user who created it.

The previous passive technique solves almost all starvation and deadlock conditions, but if a deadzock is generated by some forever blocking commands, then the technique will not work and hence requires another approach to remedy this situation.

When a tick comes, EDAK simultaneously increases the number of elapsed ticks of each ambassador object which is on duty. If the elapsed time exceeds the predefined time bound which may be determined from the current command and handler performance, $E D A K$ treats that as an abnormal situation. Thus, EDAK will try to look into the handler status. If it replies with some abnomal situation, such as no echo, pending etc, then the control hernel will recover the system for reaction. At least the control kernel will delete the current ruming command on it and, therefore, break the deadlock condition.

\subsection{Convenient Platform for Coopera- tion}

Considering a situation, if a device resource was shared by two or more handlers, then that device should have either standard interface or an agent process. For example, consider a part locating bandler which is shared by two robot arms. Because the shared handler can not be concurrently involed, therefore, $E D A K$ has to appropriately dispatch it to either of them, i.e., the robot arms. If one robot needs to grasp a part at an uncertain location, it invokes the part locating handler by issuing a cooperation request for locating decision to $E D A R$, and EDAK then selects a suitable handler (if lots of handlers meet the requirement) and ties the two handlers together. Of course, if none of suitable handlers exist in the system at that time, $E D A K$ will refuse the request and reply an error message to the robot. Or, if all of suitable handlers are busy (used by another handler) at that time, EDAK will block the request till one of suitable handlers is released, or just reply a error message back to the robot immediately. As to which of the above two strategies is suitable, it really depends on the attribute of the request command as mentioned in subsection 3.4 .

When the robot finishes the locating job, it must disconnect itself from the part locating hambler and notify $E D A K$ about the end of the relationship. Whereas EDAK takes back such handler for future management. The total flowchart is described in Fig. 4.2

But how should EDAK decides what is (are) the suitable handler(s) of this request from the robot? The answer is to look for the information recorded in the ambassador objects of the corresponding handlers during the registration phase. In the registration information, there are a number of lists which describe the characteristics of that handler, such as relationship with other handlers, access permission, supporting functions, etc. These will help to determine the meet suitable handler with respect to the request.

\subsection{Failure Detection}

Fallure detection among handlers can proceed in two ways: active or passive.

- Active : Each handler may have its own diagnosis system. When it checks itself and finds an existence of some abnormal signal, it would inform the control kernel and request to quit out actively.

- Passive: There is a patrol variable in each ambassador of a handler. This variable is cleared to zero when the corresponding handler send any normal message into the control kernel. As mentioned before, when the control kernel receives a tick, it will increase the patrol variable of every ambassador object and check whether the value of that variable exceeds a certain bound which is also predefined during the registration phase. If indeed, control kernel sends an "Are-you-up?" message to that handler that will force the handler to make a response of sending a "I-am-up" message back. Thus, it causes the patrol variable to be reset to zero again. On the contrary, if the patrolvariable is not cleared to zero and keeps increasing along with the continuous coming ticks till a certain threshold (defined in control keruel) is exceeded, then this handler is regarded as a failed one and hence is forced to quit out.

\subsection{Integrability}

The automation system controlled by EDAK can be treated as a handler also. Consequently, the higher level system designer can integrate all the subsystems using EDAK again to construct a larger system. In fact, there is a little difference between the subsystem handler and a general one. A general handler is a sequence of task executer. It can not handle more than two tasks in a duty cycle. Nevertheless, the subsystem handler is able to perform multiple tasks dispatched from the general handlers, local planners, operators, and most fascinating, its higher level system. Therefore, the subsystern handler can multiregister on multiple EDAKs. That is, the systern with any comnection network topology can be easily implemented, of which examples are depicted in Fig. 4.3. ( adopted from [8]). As a result of the above property, a hybrid control system can then be easily designed by the system designer by simply establishing relations among various subsystems.

\section{Experiment}

The experiment is performed in the two-robot assembly cell which is dedicated to assembling various types of mechanical parts serially sent in through a conveyor belt. The assembled products are then serially sent out again through the same conveyor belt to an antomated storage device. The cell is equipped with several pieces of hardware which along with their function can be described below: 
- two robots: both of them are identical in terms of their assembly function,

- conveyor belt : responsible for carrying various parts from outside into the cell and for carrying assembled products into the storage device,

- CCD cameras : form stereo vision to identify parts and to determine their configuration relative to a fixed coordinated frame,

- proximity sensors : detect the speed of each arriving part,

- assembly table and fixture: to facilitate execution of assembly tasks and to hold various sub-assemblies,

- rotary buffer : responsible for storing parts which already arrive but are not suitable for immediate assembly,

- part feeder : to supply some necessary parts (e.g., pegs) for assembly.

Each product to be assembled contains a number of parts, and all its assembly sequences which are feasible with respect to the assembly cell are assumed to be explicitly analyzed and to be stored in a knowledge base via an $A N D / O R$ graph representation $[11,12]$ as shown in Fig. 5.1. Within each assembly secquence for a type of assembly task, each assembly operation to form a sub-assembly is associated with a time cost. which is in general determined by wo factors, one is the degree of difficulty of assembly operation and the other is the cost of sensing to assist the operation.

\section{Conclusion}

We have introduced a general robotic assembly environment and the crucial portions especially in a robotic assembly cell in section 2 . The concept of the integration of handlers into a event driven antomation system is to partition the general environment into small pieces and abstract each piece as a welldefined portion of the system, with carefully defined imputs, outputs, and functions. All the commmication among handlers uniformly adopts the socket networking if a handler fails to siupport this conmmunication scheme, then an agent process is created to represent it. Such convenient communication facilitate the control kernel. EDAK, to dvannically investigate changes in the whole environment so as to let the planner conduct a precise decision-making.

Moreover, we proposed a robust procedure to build the whole system by means of the convenient ntility - EDAK classlib. Since the whole system can be view as a general handler, we can apply the same procedure to integrate various subsystems into a larger system with any kind topology and size as desired.

Besides, we build a robot assembly automation cell which is more fexible and able to real-time detecting the event of part from the conveyor belt than developed in the previonos version in the last year.

\section{References}

[1] E.T. Ho, "A rohitecture and Implementation of a Multisensor System for Robotic Assembly Environnent," M. Eng. Thesis Department of Computer Science and lnformation Engineering, NTU. 1993.

[2] Robert J. Schilling. "Fundamentals of Robotics: Analysis and Control", Prentice-Hall 1990.

[3] M. Myrup Andreasen, S. Kahler, T. Lund "T)esign for Assembly" IFS Publications, UK 1988.
(4) Cieofferey Boothroyd, Corrado Poli and Laurence E. Murch, "Automatic Assembly", Marcel Dedder, Inc. 1982

[5] Rex Maus and Randall Allsup. "Robotics : A Manager's Guide", John Wiley and Sons, Inc. 1986.

[6] Hiroshi Makino, "The Comparison Between Robotic Assembly Line and Robotic Assembly Cell", 10th International Conference on Assembly Automation, pp. 1-10, 1989.

[7] Yuan F. Zheng, "Integration of Multiple Sensors into a Robotic System and its Performance Evaluation", IEEE Journal of Robotics and Automation, Vol. 4, No. 4, pp.386396, August 1988

[8] A. Siberschatz, J. Peterson, P. Galvin "Operating System Concepts" Third edition. Addison Wesley

[9] Y. L. Chen, L. C. Fu, and T. H. Sun, "A Petri-Net Based Herarchical Structure for Dynamic Scheduler and Deadlock Avoidance," Proc of IEEE Conf. Robotics and Automat.

[10] Lj-chen Fu, and Yung-Jen Hsu, "Fully Automated TwoRobot Flexible Assembly Cell," Proc, of IEEE Conf. Robotics and Automat. 1993.

[11] L.S. Homem de Mello and A.C. Sanderson, "Representation of Mechanical Assembly Sequences," IEEE Transac. tions Robotics and Automation, Vol. 7, No. 2, pp. 221-227, 1991.

[12] L.S. Homem de Mello and A.C. Sanderson, "AND/OR Graph Representation of Assembly Plans," IEEE Transactions Robotics and Automation, Vol. 6, No. 2, pp. 188-199, 1990.705-708, 1988

[13] Cheng-Jye Luh, Bernard P. Zeigler, "Abstracting EventBased Control Models for High Autonomy Systems," IEEE Transactions on System, Man, and Cybernetics, Vol. 23, No. 1, pp. 4.20.54, 199:3

[14] M.S. Kamel, A. Thomas, "Integration of Vision, Task Planning and Robot Arm Controller," International Journal of Robotirs and Automation, Vol. 8, No. 2, 1993.

[15] Stephen T. C. Wong, "Cosmo: A Communication Scheme for Cooperative Knowledre-based System" IEEE Transactions on system, Man, and Cybernetics, Vol. 23, No. 3, ip. $809.824,1993$.

[16] (hi-Keng (Jason) Tsai "Multiple Robot Coordination and Programming," Procedangs of 1991 IEEE International Conference on Robotics and Automation, Vol. 2, pp. 978. 985

[17] Martin Boyer, Laeeque K. Daneshmend, Vincent Hayward, Andre Foisy, "An Object-Oriented Paradigm for the Design and Implementation of Robot Planning and Programming systems," Proceedings of 1991 IEEE International Conference un Robotics and Automation, Vol. $1, \mathrm{pp}$. 204-209

[18] Boyer, M., "A Knowledge-Based System for On-line Robot Error Recovery," M.Eng. Thesis, Department of Electrical Engineering, Mccill University, July 1988

[19] Meyer,B., "Object-Oriented Software Construction," Frentice-Hall, 1988

[20] Sanden, B., "The Case for Eclectic Design of Real-Time Software" IEEE Transactions on Software Engineering, Vol. 15. No.3. mp. 360.362 . March 1993.

[21] Douglas A. Bodner, Suzanne Dilley-Schneider, S. Narayanan, Uday Sreekanth, T. Govindaraj, Leon F. McGinnis and Christine M. Mitchell "Object-Oriented Modeling and Simulation of Automated Control in Manufacturing," Proceedings of 1993 IEEE International Conference on Robotics and Automation, Vol. 3, pp. 83-88. 

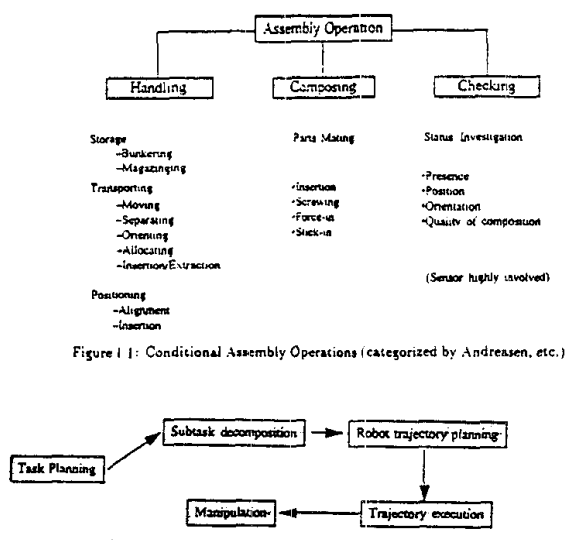

Figure 2.1: Robocic Aseemtly Control :Fow
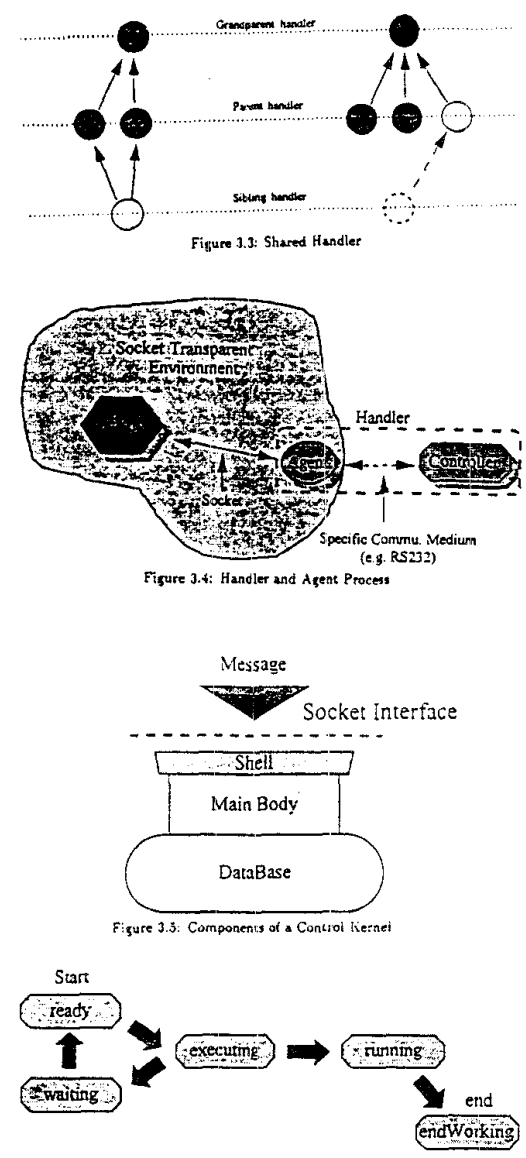

Figure 3.6: Tasi: Transition States

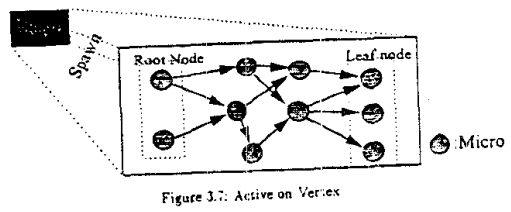

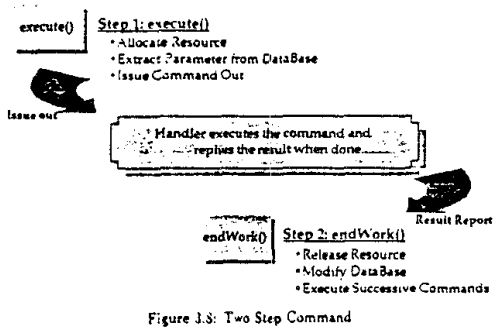

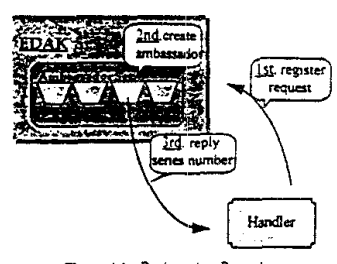

Figure 41: Registration Procedure
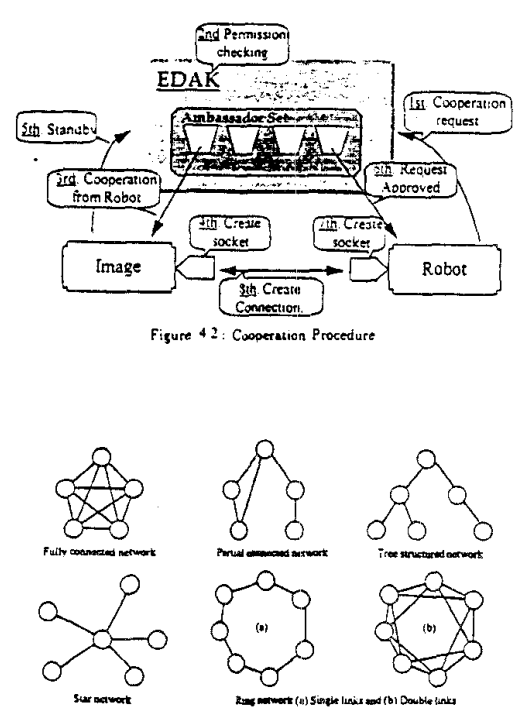

Figure 43: Connection Network

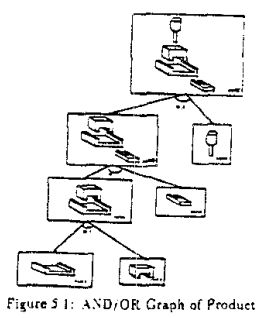

